\title{
A ROBUST DESIGN RESEARCH LANDSCAPE - REVIEW ON THE IMPORTANCE OF DESIGN RESEARCH FOR ACHIEVING PRODUCT ROBUSTNESS
}

\author{
Eifler, Tobias (1); \\ Schleich, Benjamin (2) \\ 1: Technical University of Denmark; \\ 2: Friedrich-Alexander-Universität Erlangen-Nürnberg
}

\begin{abstract}
The strive for design principles, qualitative and quantitative design tools, and product development methodologies that enable and support the design of robust products, that is products which are insensitive to noise and variation, is an ongoing challenge for both practitioners and academics. This paper reviews and classifies recent research efforts dedicated to robust design originating from the design society and carves out important challenges and potentials for future research. The main novelty of the paper can be found in the structured analysis and classification of recent robust design efforts as well as in the systematic elaboration of future research challenges, which seeks to stimulate a discussion on future tasks and challenges in robust design research.
\end{abstract}

Keywords: Robust design, Tolerance representation and management, Product structuring, Taguchi, Quality

\section{Contact:}

Eifler, Tobias

Technical University of Denmark

Engineering Design and Product Development

Denmark

tobeif@mek.dtu.dk 


\section{INTRODUCTION}

Demanding customer requirements, tightening legal regulations, and increasingly ambitious goals towards resource efficiency and economic and ecological sustainability dictate the use of sophisticated design processes and tools, which enable and support the design of robust products that are insensitive towards variations and perform their intended function even under harsh conditions and despite various fluctuating factors (Krogstie et al. 2015). This important industrial need has motivated scholars and practitioners to propose and apply various methods and tools to robust design in the past. However, while many of these methods and approaches focus on the product robustness enhancement in later design stages, the systematic and holistic design of robust products and systems in terms of conceptualisation in early design stages is often neglected, which leaves many potentials unexploited. Thus, the strive for design principles, qualitative and quantitative design tools, and product development methodologies that enable and support the design of robust products remains an ongoing challenge for both practitioners and academics. In order to tackle this challenge, several research contributions dedicated to robust design have been published in the proceedings of the ICED and DESIGN conferences during the last years.

This paper reviews and systematically classifies these latest research efforts in the design research community, and carves out important challenges and potentials for future research. In this context, the main novelty of the paper can be found in the structured analysis and classification of recent robust design efforts as well as in the systematic elaboration of future research challenges, which may point the way for the future development of robust design research.

The paper is structured as follows. The next section equips the reader with a brief introduction to robust design comprising its origins and current understanding. After that, the review and classification of current research papers is presented, followed by the discussion and conclusions derived from this classification. Thereafter, challenges and issues for future research are derived. Finally, a summary and an outlook are given.

\section{FROM QUALITY ENGINEERING TO ROBUST DESIGN METHODOLOGY}

Historically, Robust Design (RD) originates from Taguchi's Quality Engineering framework, which was proposed in the late 1950s and popularised by its implementation in the US in the 1980s (Taguchi et al., 2005). Taguchi's basic idea of an experimental robustness optimisation has thereby received most of the attention of academics as well as practitioners (Jugulum \& Frey, 2007; Hasenkamp et al., 2009) and has been extensively researched outside of the design community, summarised for example by Park et al. (2006) and Robinson et al. (2004). In contrast, design research that would allow to move $\mathrm{RD}$ efforts to earlier development phases is a lot less recognized. Examples include methods for an early assessment of product robustness by means of suitable indicators (Eifler \& Howard, 2018; Götz et al., 2020), network-topological metrics to describe the robustness of engineering systems (Haley et al., 2015), or the assessment of visual robustness (Forslund \& Söderberg, 2010). However, while this paper aims at providing an overview of these research directions, it should be noted that the basic understanding as well as the most fundamental principles still originate from Taguchi's seminal work in the late 1950 's. In order to extract the essence of robustness and to clarify the overall objectives from a design perspective, they are still the most relevant and will be discussed briefly as a basis for the presented paper.

Fundamentally, robustness describes the insensitivity of products, devices and production equipment against different sources of variation, e. g. production or assembly tolerances, not (fully) specified load scenarios or ambient use conditions (Taguchi et al., 2005). Whereas nowadays still widely accommodated by inefficiencies built into products and business processes, e. g. safety factors or disproportionate effort/costs for testing as well as for quality control measures, RD consequently aims at the development of products or processes which function as intended in spite of this variation. The underlying, essential idea of RD theory is best illustrated by means of the Quality Loss Function. Traditional quality control methods interpret all products within specification limits (SL) as equally good. In contrast, $\mathrm{RD}$ is based on the awareness that even small variations of the required product performance $\Delta \mathrm{FR}$ can lead to a loss of the customer's quality perception, see $\mathrm{L}_{\mathrm{x}}$ in Figure 1 (a). The aim of $\mathrm{RD}$ is consequently to minimize the variation of the relevant product performance, which is usually influenced by a large number of different factors. Figure 1 (b) illustrates this dependency simplified to one single design parameter (DP). The gradient of the shown so-called Transfer 
Function $F R=f(D P)$ represents the product's sensitivity towards the variation of the input parameter $\Delta \sigma_{\mathrm{DP}}$, and is basis for its robustness (Göhler et al., 2016). The steeper the gradient is, the higher the resulting variation of the performance $\Delta \sigma_{\mathrm{FR}}$ will be.

(a)

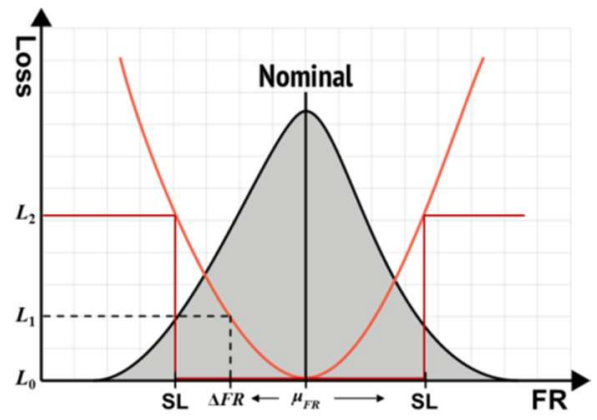

(b)

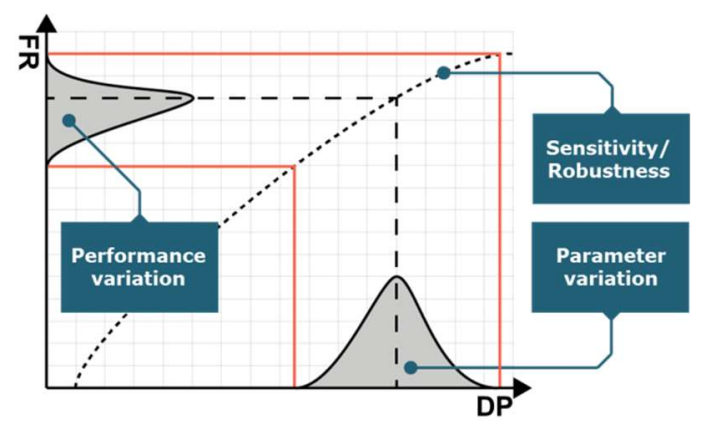

Figure 1. Relevance of variation described by a) quality loss and b) transfer function.

While the above illustrations are well-suited to explain the fundamental idea of traditional RD approaches, they at the same time illustrate the largely parametric focus as one of their main drawbacks. As shown in Figure 2, Taguchi's fundamental Quality Engineering framework advocates three different phases of a RD process, that is (1) System Design, (2) Parameter Design, and (3) Tolerance Design. While seemingly offering a comprehensive design approach, traditional RD consequently has a relatively narrow focus on one single task in embodiment design, which is the efficient optimization of parameter settings. In contrast, system design decisions such as the exploration of concepts and working principles, or the definition of suitable product configurations are largely disregarded and left to the usual design expertise. As the considered parameter settings moreover provide the basis for the allocation of tolerances in the final Tolerance Design step, the approach also excludes numerous other related Tolerancing considerations such as geometric tolerances, tolerance specifications, etc.

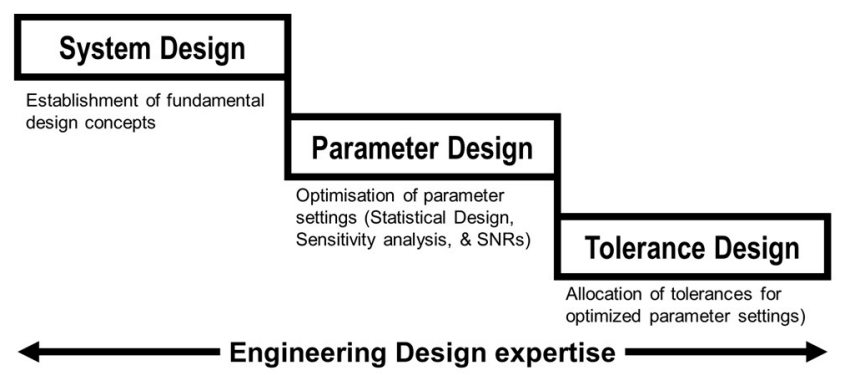

Figure 2. Three step process of Taguchi's quality engineering

As a consequence, several authors have criticized available RD for not offering enough guidance and support in early phases of development (Andersson, 1997; Jugulum \& Frey, 2007, Eifler \& Howard, 2018). Based on these insights, others have advocated to rather focus on the continuous applicability of methods and tools along the entire design process, including the tolerance design phase. The latter idea is best captured by the definition coined in Arvidsson \& Gremyr (2008), who describe the term Robust Design Methodology as:

"systematic efforts to achieve insensitivity to noise factors [where] [...] these efforts are founded on an awareness of variation and can be applied in all stages of product design"

Despite these insights on the importance of a coherent design perspective for the idea of comprehensive $\mathrm{RDM}$, it seems that RD-related research in the design community is largely scattered and not showing any specific alignment though. This includes a focus on different topics, products/systems, design phases, research methods, etc. Following the objectives formulated in the introduction, this work therefore strives to systematically map recent RD research presented at conference in the design community over the last 10 years. On this basis, the aim is to investigate focal areas of RD research with a background in design thinking, potential white spots of this research, the alignment of individual research directions and the uptake of the RDM idea, adjacent research areas, etc. 


\section{A CLASSIFICATION OF CURRENT RESEARCH CONTRIBUTIONS}

\subsection{Methodology}

In order to systematically review the ongoing research trends regarding robust design within the design society, a straightforward stepwise approach was pursued as described below. This includes a suitable query for different keywords in the design society publication database, resulting in a number of papers on robust design and related topics; the definition of a suitable portfolio classification showing relevant differences between the found papers; the manual classification according to the two portfolio dimensions; and the visualisation of results. This last step, including examples of inconsistencies that occurred during the classification as well as the necessary justification for the taken decisions is covered in the next section.

\subsubsection{Step1: Database query}

As a database, the design society homepage (https://www.designsociety.org/) was used, which provides i. a. access to the proceedings of past ICED and DESIGN conferences in a queryable manner. In this context, paper titles, abstracts, and keywords can be searched for different keywords while the relevant year, publication type, and conference series can be filtered.

Following the objectives stated in the introduction, the query was limited to papers from the last five ICED conferences (ICED11, ICED13, ICED15, ICED17, and ICED19) as well as the last five DESIGN conferences (DESIGN12, DESIGN14, DESIGN16, DESIGN18, and DESIGN20), covering the two major conferences of the design society during the last 10 years. While the material for both conference series is in general available and searchable in the database, older proceedings are only provided as a single pdf without information about the various papers. For this reason, a full text search in the conference proceedings had to be performed for the DESIGN12 conference. As search keywords, the following terms were used:

- $\quad *$ robust*, covering robust design and robustness,

- $\quad$ *reliab*, covering reliable and reliability

- $\quad *$ toleranc* ${ }^{*}$, covering tolerance (....analysis, ...management, etc.) and tolerancing

Additionally, the year and conference series were set to the corresponding conference, for example the conference series was limited to "Design" and the year to "2014" for the DESIGN14 as also illustrated in Figure 3 below.

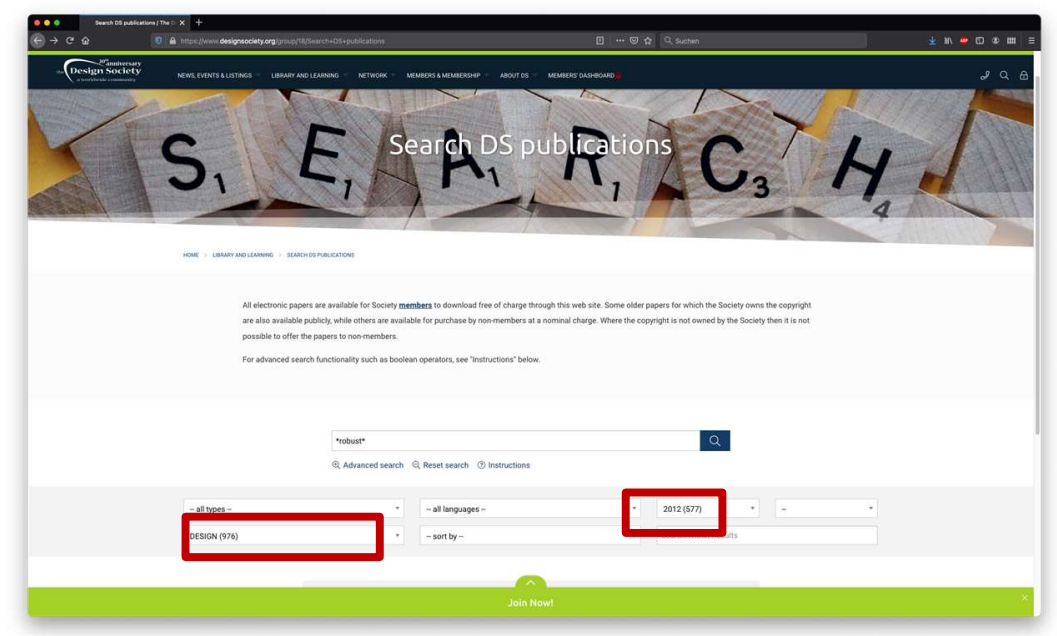

Figure 3. The homepage of the design society with relevant search filters (series and year)

As a result of the database query, 266 papers related to the area of RD were found on the basis of the figures shown in Table 1 (consider the quite large number of papers for the DESGIN12 due to the required full text search): 
Table 1. Paper review results

\begin{tabular}{l|l|l|l|l|l} 
Conference & All & $*$ robust* & $*$ reliab* & $*$ toleranc* & Unique \\
\hline ICED11 & 394 & 15 & 10 & 2 & 24 \\
DESIGN12 & 211 & 34 & 65 & 20 & 97 \\
\hline ICED13 & 351 & 11 & 12 & 5 & 25 \\
DESIGN14 & 215 & 5 & 5 & 3 & 10 \\
\hline ICED15 & 438 & 14 & 6 & 8 & 24 \\
DESIGN16 & 220 & 3 & 5 & 2 & 10 \\
\hline ICED17 & 423 & 22 & 6 & 4 & 27 \\
DESIGN18 & 271 & 5 & 3 & 4 & 9 \\
\hline ICED19 & 408 & 16 & 11 & 10 & 32 \\
DESIGN20 & 264 & 5 & 3 & 1 & 8 \\
\hline SUM & 3195 & 130 & 126 & 59 & 266
\end{tabular}

\subsubsection{Step2: Classification framework}

Based on the database query, the identified papers were classified in a suitable way. Following the overall objectives stated in section 1, the suggested classification framework has a threefold purpose:

1. provide an overview of main areas and approaches of current $\mathrm{RD}$ research;

2. to investigate how well the idea of a consistent RDM has been adopted in this research;

3. to identify potential white spots that need to be addressed in the future.

With these aspects in mind, a portfolio classification was chosen to create a research landscape along the two axes presented in Table 2. Referring to the three main phases of Taguchi's seminal Quality Engineering framework, the first axis is almost self-explanatory, and distinguishes the identified research papers according to the design phase they focus on. As discussed above, this choice implies a large simplification of the various tasks related to designing robustness into products and manufacturing systems. As it was found in the literature review that most researchers still refer to Taguchi, the use of the simplified three stage RD process, however, offers an easy to assess overview as well as a good indication how current research extents the traditional focus on the parameter design phase.

At the same time, an exclusive focus on the design phases alone will not cover the full width of actually addressed research challenges though. While there is naturally a big difference in terms of research challenges along the different phases of development, e.g. validation of qualitative evaluation results from early design in contrast to computational efficiency of variation simulation in later design, there still are large differences in the individual design phases depending on the underlying research question and the corresponding research methods. General RD literature suggests that examples could include qualitative research methods from management science (Krogstie et al., 2015, Zadeh et al., 2015), case study investigations for robustness improvements (Lönn et al., 2009), or the development of design principles based on patent research (Jugulum \& Frey, 2007).

For this reason, and to account for these differences, the framework is complemented by a second axis. First of all, this second classification thereby follows the differentiation of principles, practices, and tools, e.g. used in total quality management (Dean and Bowen, 1994) and previously adopted in the field of RD research by Hasenkamp et al. (2009). While principles are understood as capturing the key rationale for $\mathrm{RD}$ and consequently covering the question of 'why work with RD', practices are described as simple activities to fulfil the principles. Lastly, tools provide a clear step-by-step procedure, which is based on supporting methods, that provide the instructions to put the practices into action. Choosing the example of traditional Parameter Design approaches, the principle "Insensitivity to noise" is achieved by the practice "Exploit non-linearities and interactions", which in turn is put into action by crossed-array experiments and the simplified analysis based on Signal-to-noise ratios (Taguchi et al. 2015). Furthermore, this generic description of RD methodology is extended by two categories for the application of existing RD approaches as well as its industrial implementation in order to cover the often largely applied focus of RD research. A short explanation of categories can be found in Table 2 below. 
Table 2. Classification framework

\section{Principle \& \\ Practices}

Methods \& Tools

Application Cases

Implementation
Research focusing on the key rational behind robust design

and the general activities to fulfil those.

Research presenting newly developed methods and illustrating

their application or corresponding validation activities

Application of existing methods, either for product

improvements, or for method validation in a new environment.

Implementation of RD methods in an industrial organisation.

System Design $\quad$ Parameter Design $\quad$ Tolerance Design

The framework consequently classifies existing research in 12 different categories. While its first intention is to show the main focal points of RD related research, the clear definition of categories would also allow for identifying research that not only addresses one specific, but several phases or tasks in the design process, hence take up the RDM idea. Lastly, the classification exercise can also be used to investigate whether there have been significant changes of any type in the research directions seen over the last ten years.

\subsubsection{Step 3: Classification procedure}

Based on the derived framework, the papers obtained from the database query were systematically classified according to title, abstract, and keywords, keeping the overview of the corresponding conference where the work was presented. Providing a good first understanding of the research contribution in many cases, further paper details were considered when more information was necessary, for validation of the initial assignment to different categories respectively. In order to increase the reliability of results, the classification was initially performed on an individual basis by the authors. Subsequently, potential inconsistencies were discussed, which helped to avoid misunderstandings and to investigate the underlying reasons, leading to additional conclusions in many cases.

Accordingly, the classification process followed a three-step procedure. First of all, a set of papers from one single conference (ICED'13) was used as a preliminary example to ensure a common understanding of the derived classification categories. Secondly, all found papers were classified individually by the authors. Thirdly, differences in the classification were identified and discussed to align on potential disagreements. Resulting in the common, final classification presented in the following section, it goes without saying that the experienced inconsistencies between the individual classifications are equally important and are therefore considered as part of the results in section 4 .

\subsection{Classification}

Based on the initial set of 266 found papers including the search terms "*robust*", "*reliab* " "*toleranc *", the first task was to identify research that is actually relevant in the context of RD. This step resulted in a mostly overlapping identification of 82 relevant papers, 168 non-relevant papers, and 16 papers where this initial distinction of basic relevance was inconsistent. Based on the identification of adjacent research areas that had been classified differently, e. g. platform design or change management as also described in section 4, the last number could subsequently be reduced to 6 papers. In other words, in this initial step only $2,2 \%$ of the found publications required further information and discussion.

Despite all further inconsistencies in the then following step of assigning papers to different categories, the subsequent individual classification already allowed for a clear identification of key focal as well as ill-supported research areas. This is shown based on the initial, individually performed classifications shown in Figure 4 (a) and (b) below. While also discussed as part of the results in section 4, all disagreements were subsequently discussed, and solved, in an in-detailed workshop, which resulted in the final classification. This overall RD research landscape is visualised in Figure 5 on the next page and includes 79 papers classified according to the suggested 12 categories. 
(a)

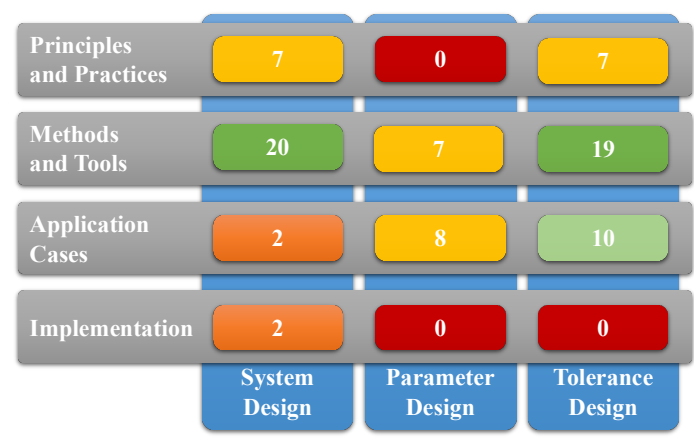

(b)

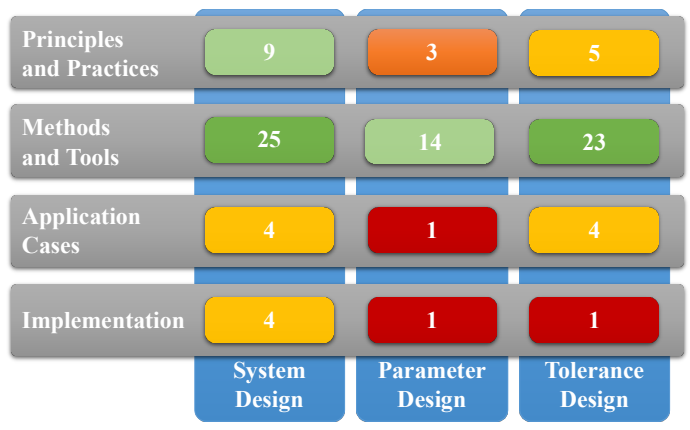

Figure 4. Initially conducted, individual classifications (a) and (b)

\section{DISCUSSION OF RESULTS}

As mentioned above, the presented classification framework offers a good basis for mapping the current RD research landscape in the design community. Not surprisingly, most research focussed on the question of method development across all phases of development. As in other areas of design research, it gets also clear that a subsequent use and validation of the developed methods stays a challenge, with significant differences though. This holds particularly true for methods suggested in the system design stage, while an application of parameter design and tolerance design methods seems to be more widespread, presumably due to the longer tradition and as the underlying quantitative models are more amendable for a typical engineering analysis, hence also easier to communicate. In all categories, there is furthermore a lack of research on the questions of method implementation in an industry context. Based on the available data, it consequently seems likely that many of the developed method and tools are not seeing a widespread application in practice or that their application is not reported in scientific literature. While the underlying reasons are certainly manifold, e. g. including typical confidentiality issues, it seems that more systematised efforts are necessary to bridge the gap between academia and industrial applications. Here, support for researchers in the design community, including for example sharing case study material with an increased focus on reproducibility of results, joint studies on contextual influences, or specific training activities for discussing the challenges of scaling individual results to a larger audience, might allow for improvements (e.g. through the DS Special Interest Groups such as https://robust.designsociety.org/).

Besides this general, potentially not this surprising, tendency towards method development in the design community, it is particularly the analysis of inconsistencies that offers interesting insights. In a first step, it was thereby possible to identify a number of research areas that are adjacent to the study of mechanical robustness and in parts use the same terminology. Examples include the robustness to expected changes (Change Management / 5 papers), robustness towards changes between product generations more specifically (Product generations \& Platforms / 7 papers), as well as the question of risk during development (Risk Management / 4 papers). In some cases, the inconsistencies during classification also allowed for identifying potential future research areas, such as the robustness and Resilience of large engineering systems (1 paper), as well as the more Technical aspects of a circular economy, e.g. repairability, diagnosis, monitoring, recycling, etc. (1 paper).

Afterwards, it was analysed how many papers were assigned inconsistently to the different categories of the suggested framework during the classification. Interestingly, only 8 of the 32 inconsistent classifications are resulting from assigning methods to different development phase. Instead, the most often occurring difference is related to the question whether research actually presents a newly developed method or tool, or whether a previously existing method is applied on a case study for testing or validation purposes.

On the one hand, this fact adds to the above-mentioned question of method development vs. method application, which once again underlines that there is often only a marginal extension of existing and well-accepted tools, and thus an often unclear research contribution. On the other hand, it also leads to a new hypothesis why this is actually the case, hence a way forward how research can be better communicated to practitioners in the future. While robustness is affected in almost all development phases, i.e. depends on conceptual decision, chosen product configurations, interface designs, the optimisation of parameter settings, or complex tolerance chains that are relevant for a multitude of life- 


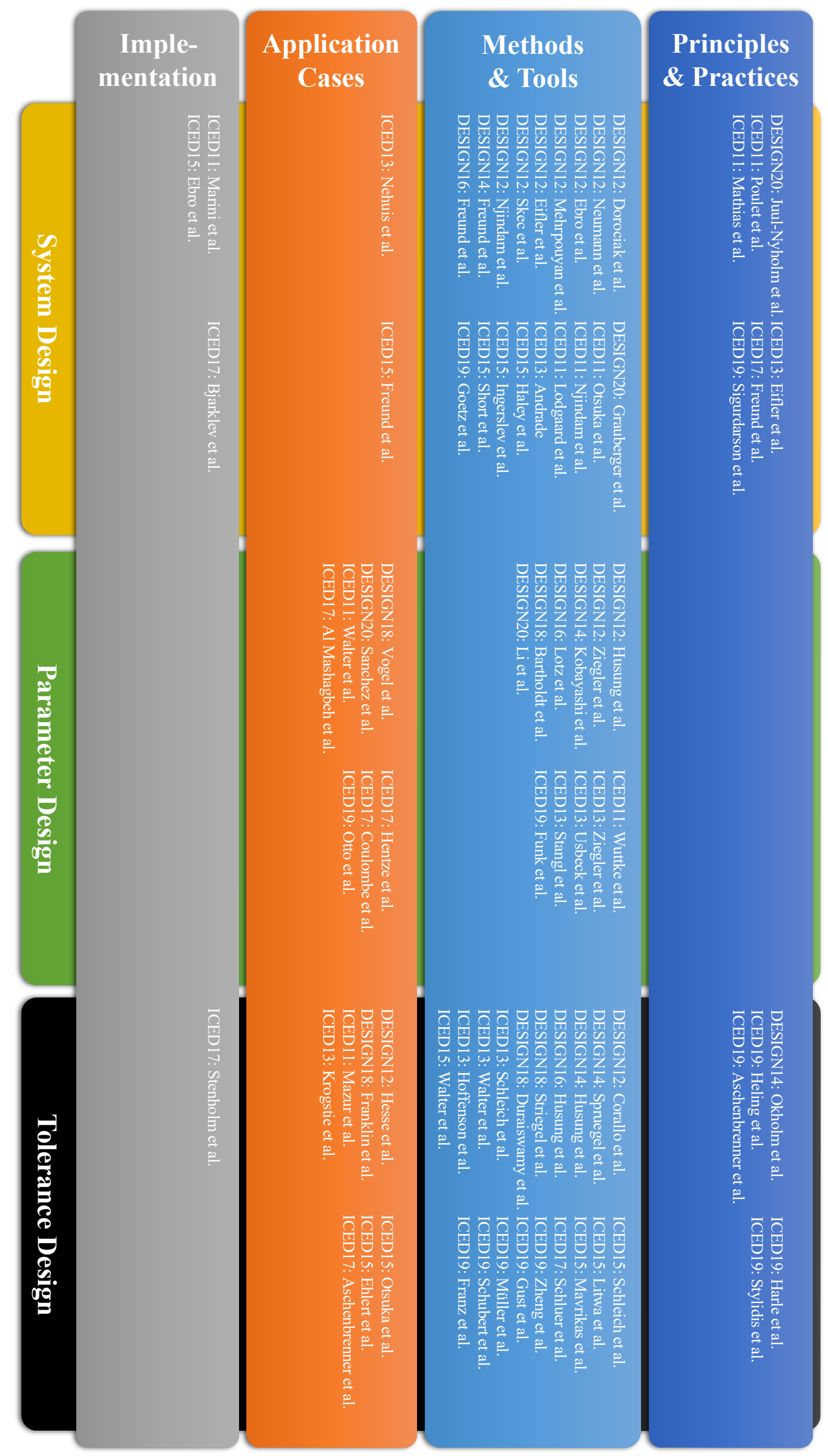

Figure 5. Final classification of RD related research ICED11 - DESIGN20 
cycle requirements, etc., current research apparently lacks a good understanding of these design tasks. Instead, most research refers either to Taguchi's simplistic three phase model, or also to simple terms such as "Early Stage" methods. In the found papers, the latter is used for a multitude of aspects, including the choice of concepts, the definition of mechanical constraints, the identification of failure modes, as well as uncertainty propagation approaches based on fuzzy variables. Moreover, it should be noted that the clear assignment of methods to the different development phases also implies that the idea of a coherent RDM, i. e. an approach for systematically to consider robustness along the entire development process, has apparently seen little adoption among academics. Work that addresses more than one development phase is rare and usually comes in a survey or review format itself, e.g. Campean et al. (2020). For this reason, it was not included in the classification of Figure 5.

Finally, the progression of topics over time is visualised in a sunburst plot in Figure 6, which is ordered according to the number of the papers in the respective company. Grouping two conferences of consecutive years to avoid any impacts of the conference structure itself, the overview shows that there has been a continuous focus on the topic RD in the design community. However, this research is of course not static. Particularly in the beginning of the reviewed period, there was a strong focus on "early stage" methods (see * in Figure 6), while the later years seem to have experienced a push towards more quantitative applications. Particularly in light of the question of the variety of RDrelated design tasks above, it should be noted though that this push seems limited to the application of existing methods in the field of parametric design. Moreover, it seems that during the last ten years, there has been a slight increase of papers dealing with the tolerance design stage, while very few papers are dealing with the systematic implementation of robust design in practice.

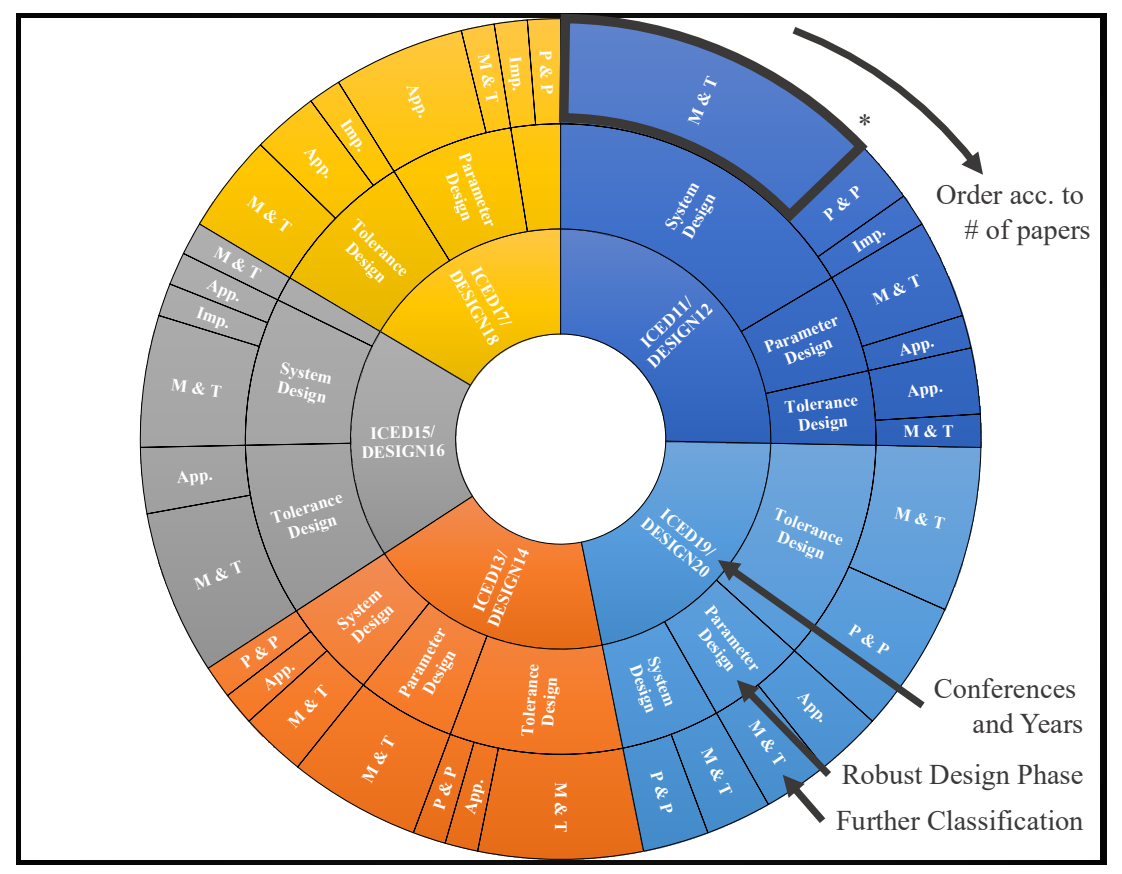

Figure 6. Research topics over time (ICED'11 - DESIGN'20), with M\&T: "Methods and tools", P\&P: "Principles and practices", App.: "Application", Imp.: "Implementation"

\section{CONCLUSION}

This paper looks at a ten years history of RD research presented at the two main conferences affiliated with the design society. The aim is to create a meaningful overview of this RD research landscape, as well as to stimulate a discussion on future research challenges for a design-driven RD approach. For this purpose, a framework was presented that allows for the systematic classification of RD-related research according to two relevant axes, and in this way also provides the basis for systematically identifying white spots in the current research landscape. Throughout the classification procedure, it was furthermore possible to identify adjacent areas of research that might be relevant in the future.

Overall, 266 papers on the topic of RD, reliability, or tolerance were identified. The classification thereby shows that research addresses mostly the development of new methods, without a clear strategy for application/validation, and without a focus on implementation in an industry context. As 
the fundamental principles and practices are neither covered extensively, this, all in all, results in the impression of largely individual methods that show no alignment across the different phases and/or task along the development process.

Particularly in light of the current, disruptive technology changes, it therefore seems almost compulsory to advance and strengthen a design-driven perspective on robustness in order to address the move from the gradual development of well-known technologies to completely new design tasks. Hence, one of the main future research potentials lies in the clarification and alignment of the various $\mathrm{RD}$ task and methods across different design phases, as well as in the systematic evaluation and strengthening of robust design method applicability in an industrial context. Furthermore, areas such as new production systems and the corresponding platform/modularity questions, the challenge of large-scale engineering systems, as well as the technical aspects of the transformation to a more circular economy model are identified as drivers for future research.

\section{REFERENCES}

Andersson, P. (1997), On Robust Design in the Conceptual Design Phase: A Qualitative Approach, Journal of Engineering Design, Vol. 8, No. 1, p. 75-89. https://doi.org/10.1080/09544829708907953.

Arvidsson, M. and Gremyr, I. (2008), Principles of Robust Design Methodology, Quality and Reliability Engineering International, Vol. 24, No. 1, p. 23-35. https://doi.org/10.1002/qre.864.

Azadeh Fazl Mashhadi, Sverker Alänge, Göran Gustafsson \& Lars-Uno Roos (2015): The Volvo Robust Engineering System: how to make robust design work in an industrial context, Total Quality Management \& Business Excellence, https://dx.doi.org/10.1080/14783363.2015.1039938

Campean, F., Delaux, D., Sharma, S., and Bridges, J. (2020). Reliability research roadmapping workshop: implications for engineering design. Proceedings of the DESIGN conference. https://dx.doi.org/10.1017/dsd.2020.337

Dean, J.W. and Bowen, D.E.: "Management Theory and Total Quality: Improving Research and Practice through Theory Development. The Academy of Management Review , Jul., 1994, Vol. 19, No. 3, Special Issue: "Total Quality" Jr. and David E. Bowen Source: The Academy of Management Review , Jul., 1994, Vol. 19, No. 3, Special Issue: "Total Quality"

Eifler, T. and Howard, T.J. (2018), The importance of robust design methodology: case study of the infamous GM ignition switch recall, Research in Engineering Design 29, 39-53 (2018). https://dx.doi.org/10.1007/s00163-017-0251-x.

Forslund, K. and Söderberg, R. (2010) "Aesthetic consequences of making car exteriors visually robust to geometrical variation", Journal of Design Research, Vol. 8, No. 3, p. 252 - 271. https://doi.org/10.1504/JDR.2010.032609

Göhler, S. M., Eifler, T., and Howard, T. J. (2016) Robustness Metrics: Consolidating the multiple approaches to quantify Robustness. Journal of Mechanical Design, Vol. 138. https://doi.org/10.1115/1.4034112.

Götz, S., Schleich, B., and Wartzack, S. (2020). "Integration of robust and tolerance design in early stages of the product development process. Research in Engineering Design, 31, 157-173. https://dx.doi.org/10.1007/s00163-019-00328-2

Haley, B.; Dong, A.; Tumer, I. (2015) "Measuring functional robustness with network topological robustness metrics", Proceedings of the 20th International Conference on Engineering Design (ICED 15), Milan/Italy 27.-30. August 2015. DOI: (if present).

Hasenkamp, T., Arvidsson, M. \& Gremyr, I. (2009) A review of practices for robust design methodology, Journal of Engineering Design, 20:6, 645-657, https://dx.doi.org/10.1080/09544820802275557.

Jugulum, R., Frey, D.D., 2007: Toward a taxonomy of concept designs for improved robustness, Journal of Engineering Design, 18:2, 139-156, https://dx.doi.org/10.1080/09544820600731496.

Krogstie, L., Ebro, M. \& Howard, T.J. (2015) How to implement and apply robust design: insights from industrial practice, Total Quality Management \& Business Excellence, 26:11-12, 1387-1405, https://dx.doi.org/10.1080/14783363.2014.934520..

Lönn, D.; Öman, M.; Nilsson, L. G.; and Simonsson, K. (2009), Finite element based robustness study of a truck cab subjected to impact loading. International Journal of Crashworthiness, Vol. 14, No. 2, p. 111-124. https://doi.org/10.1080/13588260802412992.

Park, G.J, Lee, T.H., Lee, K.W. \& Hwang, K.H. (2006) Robust Design: An Overview. AIAA JOURNAL Vol. 44, No. 1, January 2006

Robinson, T.J., Borror, C.M. \& Myers, R.H. (2004) "Robust Parameter Design - A Review”. Quality and Reliability Engineering International. 20:81-101

Taguchi, G., Chowdhury, S., and Yuin Wu B.S. (2005), “Taguchi’s Quality Engineering Handbook”. Wiley \& Sons, Inc. New Jersey. 